\title{
The Analysis of Flow Stability in Pipe by Experiment Jun WANG ${ }^{1,2, a}$, Jie GENG ${ }^{1}$, Dong LI $^{1}$, Xin-hui WANG ${ }^{2}$
}

1. School of Energy and Power Engineering, Shandong University, Jinan 250061, China

2. Shandong Jianzhu University, Jinan 250101,China

a.wwjjy99@126.com

Keywords: Velocity field, Pipe, Reynolds number, Standard deviation

Abstract: Many kinds of fluid were sent by pipes; the stability of the flow was closely related to the exchange of energy. The velocity field of a pipe was tested in the experiment; the curves of standard deviation were obtained from the test. The results show that at low Reynolds number axial and radial standard deviation curves decrease and the stabilization of the flow is better; at high Reynolds number the curves of standard deviation reach its peak at different locations; the downward trend of the curve is relatively gentle after the peak point, it has higher instability.

\section{Introduction}

Since the appearance of laser, it has been applied in many fields, and it is an important method to measure speed [1,2], we can get more information about the flow field by the application of laser velocity measuring technique. Traditional method need to decorate the speed sensor in the flow field which may have certain influence on the flow and make the error increased.

Laser emitter outside the pipe is used in Doppler velocimetry to irradiate particles in the flow field, it can get detailed informations of the flow field by characteristics of the particles[3,4]. The method will not produce interference on the flow field.

Changes in velocity have an important impact on the flow field, speed standard deviation can response the stability of the flow, standard deviation is analyzed based on the velocity field from the experiment and the flow state can be studied.

\section{Experimental Principle and Devices}

\subsection{Experimental Principle}

Laser Doppler Velocimetry uses Doppler frequency shift of scattered light by moving particles to get the velocity informations [5,6], the size and concentration of the particles such as dust in the air and impurity in the water can meet the requirements of Laser Doppler Velocimetry; particles can move with the flow of fluid better, so that we can get the details of the flow field by Laser Doppler Velocimetry.

\subsection{Experimental Devices}

The testing system [7-9] is mainly composed of laser generator, the incident light system, the receiving optical system, frequency shift device, filter device, signal processor, computer, coordinate frame system.

Incident light from the laser beam system is divided into three parallel illumination lights with different polarization properties; the receiving optical system collects scattered light emitted to the surrounding by moving particles passing through measuring area.

Electrical signal is obtained by the optical heterodyne and photoelectric conversion process. Frequency shift system determines the direction of flow and gets processible signal in the filter after amplification and filtration, the work of sampling and data processing are completed by computer software at the end.

Considering the actual situation of the flow field measurement, laser emitter, incident light unit and receiving optical unit are installed on the three-dimensional coordinate frame, it can shift the measuring point and test the cross section by the movement of optical guide rail. 


\section{Experiment System Adjustment}

\subsection{Adjustment of Incident Light System [2,10,11]}

Optical unit which has semiconductor laser is placed on the main rail.On the guide rail, the position can be moved by the sliding of rocker arm and different positions are measured.

Three beams of light emitted from the incident light unit are first order diffraction light with considerable brightness. The public beam of light should be slightly stronger than the other two, the other two should have the same brightnees. Three beams of incident light should be intersected, a magnifier is used for observing whether beams are intersected.

The calibration is carried out by a mobile magnifying glass. If the intersection of incident beams is better, image points coincide on receiving lens cap when magnifying glass is on the position of coincidece. Image point is divided into three points and presents the distribution of right angled isosceles triangle.

\subsection{Adjustment of Receiving Light System [2,10,11]}

The optical axis of the receiving unit must be located as far as possible on the same axis of the incident light unit to ensure that the receiver is at the same height with the incident light unit.

Three light points formed by incident light beam should be symmetrical distributed into a right triangle. Turning the lens cap off and openning the power of ultrasonic test device it can make particles move through the beam, the light scattered by particles could be seen in two observation mirrors of the receiving unit, adjusting lens distance fine-tuning the measuring area image can be get on the pinhole aperture plane. The image point of measuring body is directed at pinhole by regulation of displacement adjusting screw.

\section{Analysis of Experimental Result}

Change in the pipe flow is closely related to the flow stability, velocity standard deviation is an important indicator, it can describe the flow stability and volatility, the centralization and decentralization of parameters. The flow stability can be judged by analyzing the standard deviation of axial and radial velocity.

\subsection{Curves of Axial Standard Deviation}

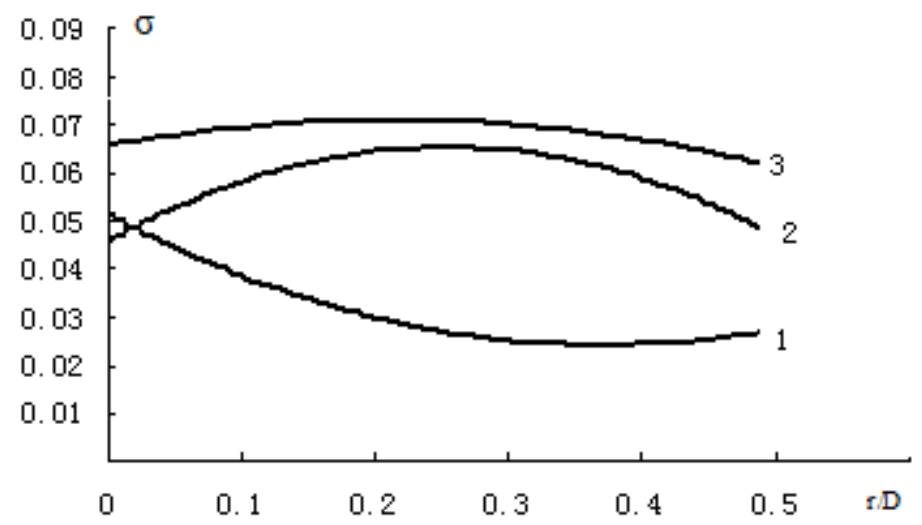

Figure. 1. Fitting curves of axial standard deviation

In order to analyze the variation of axial standard deviation, polynomial fitting method is carried out. In figure 1, curve1, 2 and 3 represent respectively the fitting curves when Reynolds number are 3000, 4000 and 5000.

When Reynolds number is 3000, the standard deviation curve has downward trend, axial flow stability is better and has fewer randomness, it reaches the bottom of the curve in $r / D=0.35$. The flow is gradually stabilized.

Curve 2 and 3 get a convex shape, fluctuations in the beginning stage have gradually developing trend,it reach their peak respectively in $\mathrm{r} / \mathrm{D}=0.26$ and $\mathrm{r} / \mathrm{D}=0.22$, after that it decreases gradually and gets stabilization situation. But the instability of curve 3 is significantly higher than that of the curve 2. In the near-wall region, the stabilization of curve 2 is greater than that of curve 3 . It can be found that the stabilization characteristic of axial standard deviation is more obvious at low 
Reynolds number and the area near the pipe wall.

\subsection{Curves of Radial Standard Deviation}

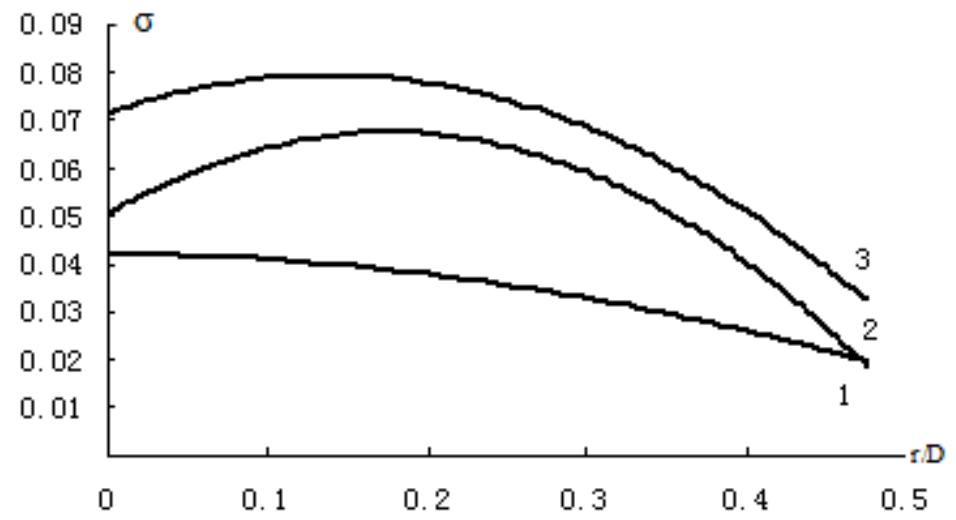

Figure. 2. Fitting curves of radial standard deviation

Figure 2 is the fitting curves of radial standard deviation fitted radial. Curve 1, 2and 3 are respectively the radial standard deviation when Reynolds number is 3000, 4000 and 5000. Although the shape of the curve 1 is convex, but the trend is downward and the shape of the curve is flat, it displays the gradually stabilized characteristics from the axis along the radial direction, the fluctuation gradually decreased.

Curve 2 and 3 are also the convex shape, they show the tendency of rising up at first and then falling down, a downward trend after the first rise, the instability of the flow is increased at first and then reduced, the difference is that curve 3 has the highest instability, it reach the peak at $r / D=0.12$, the development of the instability is fast, curve 2 get its peak at $r / D=0.18$ and its development is slow. After the peak point, both of curve 2 and 3 decrease, in contrast the declining tendency of curve 3 is more flat and has a higher unstable state. The improvement of radial instability can contribute to energy exchange between different flow layers and fluid blending.

\section{Conclusions}

1) In this paper, fluid velocity distributions of pipe flow are tested under different Reynolds number, the curves of standard deviation are obtained under different flow situation. The stability of the flow can be discriminated by the trend of standard deviation curves.

2) At low Reynolds number axial and radial standard deviation tend to decrease, the stabilization of the flow is better; at high Reynolds number standard deviation curve get downward after the first growing trend, but under different flow states the curve reach its peak at different locations, the downward trend of standard deviation is relatively gentle after the peak point, standard deviation value is greater and the flow has higer instability.

3)The stabilization characteristic of axial standard deviation is more obvious at low Reynolds number and the area near the pipe wall, the improvement of radial instability can contribute to energy exchange between different flow layers and fluid blending.

\section{References:}

[1] Principle and practice of laser Doppler testing technology. F.Durst. A.Merlin. J.H. Whitelaw. Beijing: Science Press. 1992.2

[2] Shen Xiong. Principle and application of laser doppler testing technique[M].Beijing:Tsinghua University press 2004(in chinese)

[3] Zhou Jian. Application of frequency spectrum refinementand correction technology in laser Doppler velocimeter[J].Laser\&Infrared,2010,40(2):146-151.(in Chinese)

[4] LIU You, YANG Xiao-tao, MA Xiu-zhen. Technique of flow field measurement based onlaser Doppler velocimetry.LASER \& INFRARED,Vol.42,No.1,January,2012. (in Chinese) 
[5] C. E. Willert and M. Gharib. Digital particle image velocimetry. Experiments in Fluids,1991,10:181 193.

[6] A. T. Hjelmfelt, L. F. Mockros. Motion of discrete particles in a turbulent fluid. Appl Sci Res, 1966,16: 149-161.

[7] TAKEDA Y. Velocity profile measurement by ultrasonic Doppler method [J]. Experimental Thermal Fluid Science, 1995, 10(4): 444-453.

[8] Ainsworth, R.J., Thorpe, S.J., and Manners, R.J.,New Approach to Flow-Field Measurement- a View of Doppler Global Velocimetry Techniques, International Journal of Heat and Fluid Flow, Vol. 18, No. 1, pp. 116-130, Feb., 1997.

[9] He Chun-sheng, Zhang Hong-jun,Wang Tian-yu. A Study on the Image Processing Method in Doppler Global Velocimetry [J]. Chinese Journal of Scientific Instrument. Vol.32 No.12. Dec. 2011. (in Chinese)

[10] Komine H, Brosnan S J. Instantaneous, three-component, Doppler global velocimetry[J]. Laser Anemome-try, 1991, (1):273-277.

[11] MCKENZIE R L. Measurement capabilities of planar Doppler veloeimetry using pulsed lasers[J].Applied Op-tics.1996, 35:948-64. 